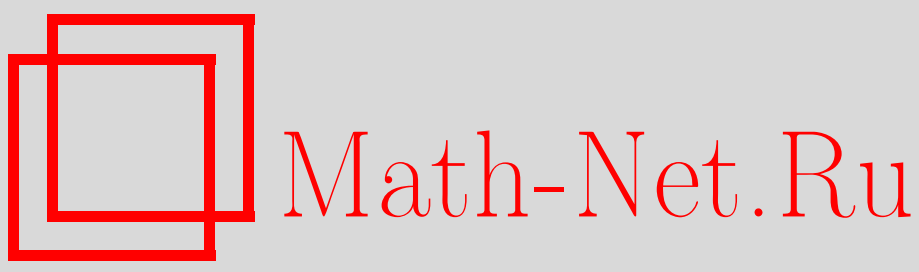

В. К. Захаров, А. В. Михалёв, Проблема общего радоновского представления для произвольного хаусдорфова пространства. II, Изв. РАН. Сер. матем., 2002, том 66, выпуск 6, 3-18

DOI: https://doi.org/10.4213/im407

Использование Общероссийского математического портала Math-Net.Ru подразумевает, что вы прочитали и согласны с пользовательским соглашением

http://www.mathnet.ru/rus/agreement

Параметры загрузки:

IP : 34.227 .88 .159

26 апреля 2023 г., 13:24:35 
УДК $517.981 .1+517.518 .1+517.982 .3$

В.К. Захаров, А.В. Михалёв

\section{Проблема общего радоновского представления для произвольного хаусдорфова пространства. II}

\footnotetext{
Проблема общего радоновского представления состоит в следующем: для хаусдорфовых топологических пространств найти пространство линейных функционалов, представимых в виде интегралов по всем радоновским мерам.

В ч. I настоящей статьи (см. [39]) получено одно из возможных решений этой проблемы. В ч. II устанавливается, что классические теоремы Рисса-Радона и Прохорова получаются как следствия теоремы об общем интегральном радоновском представлении, доказанной в [39].

Библиография: 39 наименований.
}

После основополагающих работ [1]-[3] стала актуальной проблема общего радоновского представления. Суть ее состоит в следующем. На хаусдорфовом топологическом пространстве $(T, \mathscr{G})$ рассматривается семейство $\mathfrak{R M}^{w o}(T, \mathscr{G})_{0}$ всех положительных широких борелевско-радоновских мер $\mu$ из $\mathscr{B}(T, \mathscr{G})$ в $[0, \infty]$. Каждая мера $\mu$ порождает интегральный радоновский функиионал $i_{\mu} \equiv \int \cdot d \mu$ на векторном пространстве $\mathrm{MI}(T, \mathscr{B}(T, \mathscr{G}), \mu)$ всех $\mathscr{B}(T, \mathscr{G})$-измеримых $\mu$-интегрируемых функций $f$ из $T$ в $\mathbb{R}$.

Все радоновские функционалы $i_{\mu}$ можно рассматривать на любом векторном пространстве $A(T)$, лежащем в векторном пространстве

$$
\mathrm{UI}(T) \equiv \bigcap\left(\mathrm{MI}(T, \mathscr{B}(T, \mathscr{G}), \mu) \mid \mu \in \mathfrak{R M}^{w o}(T, \mathscr{G})_{0}\right)
$$

всех $\mathscr{B}(T, \mathscr{G})$-измеримых универсально-интегрируемых функций относительно всех указанных радоновских мер таком, что отображение сужения $\mu \mapsto i_{\mu} \mid A(T)$ из множества $\mathfrak{R M} \mathfrak{M o}^{w o}(T, \mathscr{G})_{0}$ в векторное пространство $A(T)^{\times}$всех линейных функционалов на $A(T)$ является инъективным. Поэтому возникает проблема абстрактного описания семейства всех интегральных радоновских функиионалов (обычно именуемая как проблема интегрального радоновского представления): для некоторого подходящего векторного пространства $A(T)$ в векторном пространстве $A(T)^{\times}$всех линейных функционалов на нем выделить в абстрактных (т.е. не используюших меры) терминах некоторое векторное решеточное подпространство $A(T)^{\triangle}$ такое, что его положительная часть $\left(A(T)^{\Delta}\right)_{+}$в точности совпадает с семейством $A(T)_{0}^{r f} \equiv\left\{i_{\mu}|A(T)| \mu \in \mathfrak{R M}^{w o}(T, \mathscr{G})_{0}\right\}$ всех положительных радоновских функционалов на $A(T)$.

Рассматривается также ограниченная версия этой проблемы (проблема ограниченного интегрального радоновского представления), когда вместо семейства $A(T)_{0}^{r f}$ рассматривается векторное пространство $A(T)_{b}^{r f} \equiv\left\{i_{\mu}|A(T)| \mu \in\right.$ $\left.\mathfrak{R M}_{b}^{w o}(T, \mathscr{G})\right\}$ всех равномерно ограниченных радоновских функционалов, соответствующих всем ограниченным радоновским мерам $\mu$ из $\mathscr{B}(T, \mathscr{G})$ в $[a, b] \subset \mathbb{R}$.

(C) В. К. ЗАХАРов, А.В. МИхАлЁв, 2002 
Решение проблемы радоновского представления для случая локально компактного пространства было дано Халмошем [7], Хьюитом [8] и Эдвардсом [9] и известно как общая теорема Рисса-Радона о представлении (см., например, [11] или [12]).

Теорема. Пусть $(T, \mathscr{G})$ - локально компактное пространство и $C_{c}(T, \mathscr{G})$ векторное решеточное пространство всех непрерывных функций на $(T, \mathscr{G}) c$ компактными носителями. Тогда:

1) отображение сужения $\mu \mapsto i_{\mu} \mid C_{c}(T, \mathscr{G})$ из $\mathfrak{R M}^{w o}(T, \mathscr{G})_{0}{ }_{\boldsymbol{\theta}} C_{c}(T, \mathscr{G})^{\times}$ является инғективным;

2) семейство $C_{c}(T, \mathscr{G})_{0}^{r f}$ всех положительных радоновских функционалов в точности совпадает с положительной частью $\left(C_{c}(T, \mathscr{G})^{\sim}\right)_{+}$векторного решеточного пространства всех линейных бинарно-положительных функционалов на $C_{c}(T, \mathscr{G})$.

Решение проблемы ограниченного радоновского представления для случая тихоновского пространства было дано Прохоровым [13].

ТЕОРемА. Пусть $(T, \mathscr{G})$ - тихоновское пространство и $C_{b}(T, \mathscr{G})$ - векторное решеточное пространство всех непрерывных ограниченных функиий на $(T, \mathscr{G})$. Тогда:

1) отображение ограниченного сужения $\mu \mapsto i_{\mu} \mid C_{b}(T, \mathscr{G})$ из $\mathfrak{R M}_{b}^{w o}(T, \mathscr{G})$ в $C_{b}(T, \mathscr{G})^{\times}$является инвективнылм;

2) решеточное векторное пространство $C_{b}(T)_{b}^{r f}$ всех равномерно ограниченных радоновских функционалов в точности совпадает с векторным решеточным пространством $\left(C_{b}(T, \mathscr{G})\right)^{(\pi)}$ всех линейных узких бинарно-положительных равномерно ограниченных функционалов на $C_{b}(T, \mathscr{G})$.

Одно из возможных решений проблемы радоновского представления для общего случая произвольного хаусдорфова пространства было дано авторами настоящей статьи (см. [21]-[23] и [39]).

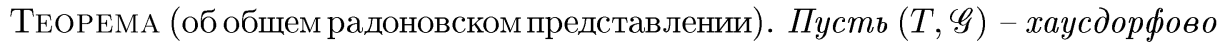
пространство, $S(T, \mathscr{G})$ - векторное решеточное пространство всех метаполунепрерывных (ограниченных) функиийна $(T, \mathscr{G})$ и $S_{c}(T, \mathscr{G})$ - coответствующее подпространство функций с компактными носителями. Тогда:

A.1) отображсение сужения $\mu \mapsto i_{\mu} \mid S_{c}(T, \mathscr{G})$ из $\mathfrak{R M}^{w o}(T, \mathscr{G})_{0}$ в $S_{c}(T, \mathscr{G})^{\times}$ является инбективным; 2) семейство $S_{c}(T, \mathscr{G})_{0}^{r f}$ всех положительных радоновских функиионалов в точности совпадает с положительной частью $\left(S_{c}(T, \mathscr{G})^{\Delta}\right)_{+}$векторного решеточного пространства всех линейньх тонких бинарно-положстельных функиионалов на $S_{c}(T, \mathscr{G})$;

B.1) отображсние ограниченного сужения $\mu \mapsto i_{\mu} \mid S(T, \mathscr{G})$ из $\mathfrak{R M}_{b}^{w o}(T, \mathscr{G})$ в $S(T, \mathscr{G})^{\times}$является ингективным; 2) решеточное векторное пространство $S(T, \mathscr{G})_{b}^{r f}$ всех равномерно ограниченных радоновских функционалов в точности совпадает с векторным решеточным пространством $S(T, \mathscr{G})^{\ominus}$ всех линейных тонких бинарно-положстельных равномерно ограниченных функционалов на $S(T, \mathscr{G})$.

После выхода статей [21]-[23] и [39] оставался открытым вопрос: следуют ли из теоремь об общем радоновском представлении теоремь Рисса-Радона u Прохорова? В настоящей статье дается положительный ответ на этот вопрос: 
теоремы Рисса-Радона и Прохорова следуют из теоремы об общем радоновском представлении (см. теоремы о следствии).

Настоящая статья является непосредственньм продолжением статьи [39], поэтому за всеми необходимыми сведениями мы отсылаем к ней. Более того, мы продолжаем нумерацию пунктов $\S 2$ статьи [39].

Авторы выражают благодарность профессору А. В. Яковлеву за плодотворные обсуждения, результатом которых явилась настояшая работа.

6. Теорема о следствии для локально компактного пространства. В этом пункте покажем, что теорема Рисса-Радона для локально компактного пространства следует из теоремы об общем радоновском представлении для хаусдорфова пространства.

Пусть $(T, \mathscr{G})$ - локально компактное пространство и $\varphi$ - линейный положительный функционал на $C_{c}(T, \mathscr{G})$, т.е. $\varphi \in\left(C_{c}(T, \mathscr{G})^{\sim}\right)_{+}$. Покажем, что функционал $\varphi$ продолжается единственным образом до линейного положительного тонкого функционала на $S_{c}(T, \mathscr{G})$, т.е. $\varphi \in\left(S_{c}(T, \mathscr{G})^{\Delta}\right)_{+}$.

Пусть $A(T)$ - произвольное подмножество в $F(T)$. Напомним, что функционал $\xi$ из $A(T)$ в $\mathbb{R}$ называется поточечно непрерьвныцм, если $f=p-\lim \left(f_{m} \mid m \in M\right)$ влечет $\xi f=\lim \left(\xi f_{m} \mid m \in M\right)$ для каждой монотонной сети $\left(f_{m} \in A(T) \mid m \in M\right)$ и каждой функции $f \in A(T)$. Если $A(T)$ - решеточное векторное пространство, то это свойство равносильно каждому из следуюших свойств:

$1)$ условие $\left(f_{m} \in A(T)_{+} \mid m \in M\right) \downarrow 0$ в $F(T)$ влечет условие $\left(\xi f_{m} \mid m \in M\right) \downarrow 0$ в $\mathbb{R}$;

2) условия $f \in A(T)$ и $\left(f_{m} \in A(T)_{+} \mid m \in M\right) \uparrow f$ в $F(T)$ влекут условие $\left(\xi f_{m} \mid m \in M\right) \uparrow \xi f$ в $\mathbb{R}$.

Аналогичная равносильность свойств 1) и 2) справедлива для поточечной $\sigma$-непрерывности по последовательностям $\left(f_{m} \in A(T) \mid m \in M \subset \omega\right)$.

Следуюшая лемма является ключевой для дальнейших рассуждений, поэтому приводим ее с доказательством, хотя она является хорошо известной (см. [12, п. $73 \mathrm{D}])$.

ЛЕмма 1. Функиионал $\varphi$ на $C_{c}(T, \mathscr{G})$ является поточечно непрерывным.

ДокАЗАТЕЛЬСТво. Пусть $\left(f_{m} \in C_{c}(T, \mathscr{G})_{+} \mid m \in M\right) \downarrow 0$ в $F(T)$. Зафиксируем $m_{0} \in M$ и рассмотрим функцию $g \equiv f_{m_{0}}$ и компактное множество $C \equiv \operatorname{cl}\{t \in T \mid g(t)>0\}$. Для $C$ существует функция $h \in C_{c}(T)_{+}$такая, что $h \geqslant \chi(C)$.

Возьмем $\varepsilon>0$. Тогда найдется $a>0$ такое, что $a \varphi(h)<\varepsilon$. Рассмотрим открытые множества $G_{m} \equiv\left\{t \in T \mid f_{m}(t)<a\right\}$. Так как $\left(f_{m} \mid m \in M\right) \downarrow 0$, то $\left(G_{m} \mid m \in M\right) \uparrow T$. Поэтому существует конечное покрытие $\left(G_{n} \mid n \in N \subset M\right)$ множества $C$. Из направленности $M$ следует, что существует $m \in M$ такое, что $n \leqslant m$ для любого $n \in N$. Поэтому $G_{m} \supset G_{n}$ для любого $n$. Значит, $C \subset G_{m}$. Возьмем $l \in M$ такое, что $l \geqslant m_{0}$ и $l \geqslant m$. Тогда $f_{l} \leqslant g$ и $f_{l} \leqslant f_{m}$. Из первого неравенства следует, что $H \equiv \operatorname{coz} f_{l} \subset C \subset G_{\mu}$, а из второго неравенства следует, что $f_{l}(t) \leqslant f_{m}(t)<a=a \chi(C)(t) \leqslant a h(t)$ для любой точки $t \in H$. Значит, $f_{l} \leqslant a h$ влечет $\varphi f_{l} \leqslant a \varphi(h)<\varepsilon$. Если $k<l$, то $\varphi f_{k} \leqslant \varphi f_{l}<\varepsilon$. Таким образом, $\left(\varphi f_{m} \mid m \in M\right) \downarrow 0$. Лемма доказана.

Используя свойство поточечной непрерывности функционала $\varphi$, будем строить его продолжение некоторым упрошенным вариантом метода Даниэля, описанного 
в [29]. Приведем необходимые нам сведения из [29], опуская их доказательство.

Рассмотрим множество $S\left(T, C_{c}(T, \mathscr{G})\right)$ всех функций $g \in F(T)$ таких, что $g=$ $\sup \left(f_{m} \in C_{c}(T, \mathscr{G}) \mid m \in M\right)$ в $F(T)$ для некоторой сети $\left(f_{m} \in C_{c}(T, \mathscr{G}) \mid m \in\right.$ $M) \uparrow$ и $g \leqslant f$ для некоторой функции $f \in C_{c}(T, \mathscr{G})$. Такие функции будем называть супремальныци для $C_{c}(T, \mathscr{G})$. Аналогичным образом рассмотрим множество $I\left(T, C_{c}(T, \mathscr{G})\right)$ всех функций $h \in F(T)$ таких, что $h=\inf \left(f_{m} \in C_{c}(T, \mathscr{G}) \mid m \in\right.$ $M)$ в $F(T)$ для некоторой сети $\left(f_{m} \in C_{c}(T, \mathscr{G}) \mid m \in M\right) \downarrow$ и $h \geqslant f$ для некоторой функции $f \in C_{c}(T, \mathscr{G})$. Такие функции будем называть инфимальными для $C_{c}(T, \mathscr{G})$. Ясно, что $C_{c}(T, \mathscr{G})=S\left(T, C_{c}(T, \mathscr{G})\right) \cap I\left(T, C_{c}(T, \mathscr{G})\right)$.

Далее временно будем обозначать $S\left(T, C_{c}(T, \mathscr{G})\right)$ и $I\left(T, C_{c}(T, \mathscr{G})\right)$ через $Y$ и $Z$ соответственно.

Лемма 2. Множества $Y$ и $Z$ относительно операций в $F(T)$ обладают следующими свойствами:

1) $\sum\left(a_{i} g_{i} \mid i \in I\right) \in Y$ u $\sum\left(a_{i} h_{i} \mid i \in I\right) \in Z$ для любых конечных коллекиий $\left(a_{i} \in \mathbb{R}_{+} \mid i \in I\right), \quad\left(g_{i} \in Y \mid i \in I\right) u\left(h_{i} \in Z \mid i \in I\right)$;

$2) \inf \left(g_{i} \mid i \in I\right) \in Y$ u $\sup \left(h_{i} \mid i \in I\right) \in Z$ для любых конечных коллекиий $\left(g_{i} \in Y \mid i \in I\right) u\left(h_{i} \in Z \mid i \in I\right)$;

3) $\sup \left(g_{i} \mid i \in I\right) \in Y u \inf \left(h_{i} \mid i \in I\right) \in Z$ для любых коллекиий $\left(g_{i} \in Y \mid i \in I\right)$ $u\left(h_{i} \in Z \mid i \in I\right)$ и любых функиий $g \in Y$ u $h \in Z$ таких, что $g_{i} \leqslant g u h_{i} \geqslant h$ при всех $i$;

4) $g \wedge \mathbf{1} \in Y, g \vee(-\mathbf{1}) \in Y, \quad h \wedge \mathbf{1} \in Z u h \vee(-\mathbf{1}) \in Z$ для любых функиий $g \in Y u h \in Z$

5) $-Y \subset Z u-Z \subset Y$, m.e. $-g \in Z u-h \in Y$ для любих $g \in Y u h \in Z$.

Определим на $Y$ вещественный функционал $\bar{\varphi}$, полагая $\bar{\varphi} g \equiv \sup \{\varphi f \mid f \in$ $\left.C_{c}(T, \mathscr{G}) \wedge f \leqslant g\right\}$ для каждого $g \in Y$. Аналогичным образом определим на $Z$ вещественный функционал $\underline{\varphi}$, полагая $\underline{\varphi} h \equiv \inf \left\{\varphi f \mid f \in C_{c}(T, \mathscr{G}) \wedge f \geqslant h\right\}$ для каждого $h \in Z$. Ясно, что $\bar{\varphi}$ и $\underline{\varphi}$ являются продолжениями $\varphi$.

С помошью леммы 1 доказывается

Лемма 3. Если $g \in Y u\left(f_{m} \mid m \in M\right) \uparrow g$ для некоторой сети $\left(f_{m} \in\right.$ $\left.C_{c}(T, \mathscr{G})_{+} \mid m \in M\right), m o\left(\varphi f_{m} \mid m \in M\right) \uparrow \bar{\varphi} g$.

ЛЕмма 4. Функционал $\bar{\varphi}$ на множестве $Y$ и функционал $\varphi$ на множестве $Z$ обладают следующими свойствами:

1) $\bar{\varphi}$ и $\varphi$ возрастают;

2) $\bar{\varphi}\left(\sum\left(a_{i} g_{i} \mid i \in I\right)\right)=\sum\left(a_{i} \bar{\varphi} g_{i} \mid i \in I\right) u \underline{\varphi}\left(\sum\left(a_{i} h_{i} \mid i \in I\right)\right)=\sum\left(a_{i} \underline{\varphi} h_{i} \mid\right.$ $i \in I)$ для любых конечных коллекций $\left(a_{i} \in \overline{\mathbb{R}}_{+} \mid i \in I\right), \quad\left(g_{i} \in Y \mid i \in I\right)$ u $\left(h_{i} \in Z \mid i \in I\right)$;

3) $\left(\bar{\varphi}\left(g_{m}\right) \mid m \in M\right) \uparrow \bar{\varphi} g$ для любой сети $\left(g_{m} \in Y \mid m \in M\right)$ и любой функиии $g \in Y$ такой, чтоо $\left(g_{m} \in Y \mid m \in M\right) \uparrow g$ в $F(T)$;

4) $\underline{\varphi}(-g)=-\bar{\varphi}(g)$ u $\bar{\varphi}(-h)=-\underline{\varphi}(h)$ для любых функиий $g \in Y$ и $h \in Z$;

5) $\underline{\varphi} v \leqslant \bar{\varphi} u \quad u \bar{\varphi} u \leqslant \underline{\varphi} w$ для любых функиий $u \in Y u v, w \in Z$ таких, ито $v \leqslant u \leqslant w$.

Определим теперь на множестве $F_{b c}(T)$ всех вещественных ограниченных функций с компактными носителями вешественные функционалы $\widetilde{\varphi}$ и $\underset{\sim}{\varphi}$, полагая $\widetilde{\varphi} f \equiv$ $\inf \{\bar{\varphi} g \mid g \in Y \wedge g \geqslant f\}$ и $\underline{\sim} f \equiv \sup \{\underline{\varphi} h \mid h \in Z \wedge h \leqslant f\}$ для каждой функции $f \in F_{b c}(T)$. Это определение корректно. Действительно, так как функция $f$ ограничена и имеет компактный носитель, то сушествуют компактное множество $C$ и 
число $a>0$ такие, что $|f| \leqslant a \chi(C)$. Поэтому $|f| \leqslant a \chi(C) \leqslant f_{0}$ для некоторой функции $f_{0} \in C_{c}(T, \mathscr{G})$. В результате $\widetilde{\varphi} f \geqslant \varphi\left(-f_{0}\right)>-\infty$ и $\varphi f \leqslant \varphi f_{0}<\infty$. Ясно, что $\widetilde{\varphi}$ является продолжением $\bar{\varphi}$, а $\underline{\sim}$ является продолжением $\underline{\varphi}$.

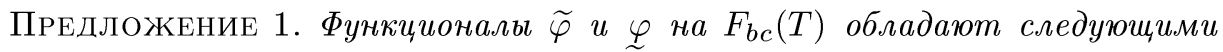
свойствами:

1) $\widetilde{\varphi}$ и $\underset{\varphi}{\varphi}$ возрастают;

2) $\varphi f \widetilde{\sim} \widetilde{\varphi} f$ для любого $f \in F_{b c}(T)$;

3) $\widetilde{\widetilde{\varphi}}(-f)=-\varphi f$ u $\varphi(-f)=-\widetilde{\varphi} f$ для любого $f \in F_{b c}(T)$;

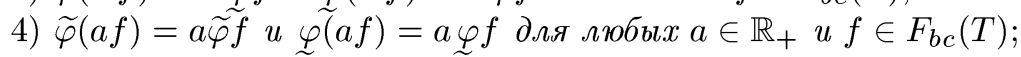

5) $\widetilde{\varphi}\left(f^{\prime}+f^{\prime \prime}\right) \leqslant \widetilde{\varphi} f^{\prime}+\widetilde{\varphi} f^{\prime \prime}$ u $\underset{\varphi}{\varphi}\left(f^{\prime}+f^{\prime \prime}\right) \geqslant \underline{\varphi} f^{\prime}+\varphi f^{\prime \prime}$ для любых $f^{\prime}, f^{\prime \prime} \in F_{b c}(T)$;

6) $\left(\widetilde{\varphi} f_{n} \mid n \in \mathbb{N}\right) \uparrow \widetilde{\varphi} f$ для любой последовательности $\left(f_{n} \in F_{b c}(T) \mid n \in \mathbb{N}\right)$ и любой функиии $f \in F_{b c}(T)$ такой, что $\left(f_{n} \mid n \in \mathbb{N}\right) \uparrow f$ в $F(T)$.

Функцию $f \in F(T)$ назовем даниелевской относительно семейства $C_{c}(T, \mathscr{G})$ $u$ функционала $\varphi$, если для любого $\varepsilon>0$ существуют функции $g \in S\left(T, C_{c}(T, \mathscr{G})\right)$ и $h \in I\left(T, C_{c}(T, \mathscr{G})\right)$ такие, что $h \leqslant f \leqslant g$ и $\bar{\varphi} g-\underline{\varphi} h<\varepsilon$. Семейство всех таких функций обозначим через $D\left(T, C_{c}(T, \mathscr{G}), \varphi\right)$. Ясно, что $D\left(T, C_{c}(T, \mathscr{G}), \varphi\right)=\{f \in$ $\left.F_{b c}(T) \mid \widetilde{\varphi} f=\underline{\sim} f\right\}$. Определим вещественный функционал $\widehat{\varphi}$ на $D\left(T, C_{c}(T, \mathscr{G}), \varphi\right)$, положив $\widehat{\varphi} f \equiv \widetilde{\varphi} f=\underline{\sim} f$. Далее иногда вместо $D\left(T, C_{c}(T, \mathscr{G}), \varphi\right)$ будем писать просто $X_{\varphi}$.

С помошью предыдуших утверждений доказывается следующая

TEОРема 1. Пусть $(T, \mathscr{G})$ - локально компактное пространство и $\varphi \in$ $\left(C_{c}(T)^{\sim}\right)_{+} \cdot$ Tогдa:

1) семейство $D\left(T, C_{c}(T, \mathscr{G}), \varphi\right)$ является векторным решеточным пространством;

2) $S\left(T, C_{c}(T, \mathscr{G})\right) \cup I\left(T, C_{c}(T, \mathscr{G})\right) \subset D\left(T, C_{c}(T, \mathscr{G}), \varphi\right)$;

3) функиионал $\widehat{\varphi}$ является линейным положительным продолжением функиионала $\varphi$;

4) функционал $\widehat{\varphi}$ является поточечно б-непрерывным.

Докажем теперь, что $S_{c}(T, \mathscr{G}) \subset D\left(T, C_{c}(T, \mathscr{G}), \varphi\right)$.

Далее через $\mathscr{G}_{c}$ будем обозначать множество всех открытых предкомпактных множеств из $T$.

ЛЕмма 5. Пусть $(T, \mathscr{G})$ - локально компактное пространство и $A \in$ $\mathscr{A}_{c}(T, \mathscr{G})$. Тогда $A=\bigcup\left(C_{i} \cap D_{i} \mid i \in I\right)$ для некоторых конечных коллекиий $\left(C_{i} \in \mathscr{C} \mid i \in I\right)$ u $\left(D_{i} \in \mathscr{G}_{c} \mid i \in I\right)$.

ДоКАЗАТЕЛЬСТво. По условию $A=\bigcup\left(F_{i} \cap G_{i} \mid i \in I\right) \subset C$ для некоторых конечных коллекций $\left(F_{i} \in \mathscr{F} \mid i \in I\right)$ и $\left(G_{i} \in \mathscr{G} \mid i \in I\right)$ и некоторого компактного множества $C$. Так как $T$ локально компактно, то существует открытое предкомпактное множество $H$ такое, что $C \subset H$. Поэтому

$$
F_{i} \cap G_{i}=F_{i} \cap G_{i} \cap C \cap H=\left(F_{i} \cap C\right) \cap\left(G_{i} \cap H\right) .
$$

Рассмотрим множества $C_{i} \equiv F_{i} \cap C$ и $D_{i} \equiv G_{i} \cap H$. Тогда $A=\bigcup\left(C_{i} \cap D_{i} \mid i \in I\right)$.

Лемma 6. Пусть $A \in \mathscr{A}_{c}(T, \mathscr{G})$. Тогда $\chi(A) \in D\left(T, C_{c}(T, \mathscr{G}), \varphi\right)$. 
ДокАЗАТЕЛЬСТво. Если $D$ - открытое предкомпактное множество, то $g \equiv$ $\chi(D) \in Y \subset X_{\varphi}$. Если $C$ - компактное множество, то $h \equiv \chi(C) \in Z \subset X_{\varphi}$. Так как $X_{\varphi}$ - решетка, то $\chi(C \cap D)=g \wedge h \in X_{\varphi}$.

По лемме $5 \quad A=\bigcup\left(C_{i} \cap D_{i} \mid i \in I\right)$. Поэтому $\chi(A)=\sup \left(\chi\left(C_{i} \cap D_{i}\right) \mid i \in\right.$ $I) \in X_{\varphi}$.

ПРЕДЛОЖЕНИЕ 2. $S_{c}(T, \mathscr{G}) \subset D\left(T, C_{c}(T, \mathscr{G}), \varphi\right)$.

ДокаЗАТЕЛЬСТво. Пусть $f \in S_{c}$. Возьмем любое $\varepsilon>0$. При доказательстве теоремы 1 из [39, $\S 2$, п. 4] было установлено, что для $\varepsilon$ существует ступенчатая функция $u \in \operatorname{St}(T, \mathscr{A}(T, \mathscr{G}))$ такая, что $|f(t)-u(t)|<\varepsilon / 4$ для всех $t \in T$. Возьмем $g^{\prime} \equiv u-(\varepsilon / 4) \mathbf{1}$ и $h^{\prime} \equiv u+(\varepsilon / 4) \mathbf{1}$. Тогда

$$
\begin{aligned}
& g^{\prime}(t)=(u(t)-f(t))+f(t)-\varepsilon / 4 \leqslant f(t), \\
& h^{\prime}(t)=(u(t)-f(t))+f(t)+\varepsilon / 4 \geqslant f(t) .
\end{aligned}
$$

С другой стороны,

$$
\begin{aligned}
& g^{\prime}(t)=(u(t)-f(t))+f(t)-\varepsilon / 4>f(t)-\varepsilon / 2, \\
& h^{\prime}(t)=(u(t)-f(t))+f(t)+\varepsilon / 4<f(t)+\varepsilon / 2 .
\end{aligned}
$$

Поскольку функция $f$ ограничена и имеет компактньй носитель, то существуют компактное множество $C$ и числа $a$ и $b$ такие, что $a \chi(C) \leqslant f \leqslant b \chi(C)$. Обозначим $\chi(C)$ через $x$ и рассмотрим ступенчатые функции с компактными носителями $v \equiv$ $\left(a x \vee g^{\prime}\right) \wedge b x$ и $w \equiv\left(a x \vee h^{\prime}\right) \wedge b x$. Тогда

$$
f(t)-\varepsilon / 2<v(t) \leqslant f(t) \leqslant w(t)<f(t)+\varepsilon / 2
$$

для любого $t \in T$ и $0 \leqslant w-v \leqslant \varepsilon x$.

Так как $v \in \operatorname{St}(T, \mathscr{A}(T, \mathscr{G}))$, то $v=\sum\left(a_{k} \chi\left(A_{k}\right) \mid k \in K\right)$ для некоторых коллекций $\left(a_{k} \in \mathbb{R} \mid k \in K\right)$ и $\left(A_{k} \in \mathscr{A}(T, \mathscr{G}) \mid k \in K\right)$. В силу того, что $\mathscr{A}(T, \mathscr{G})-$ алгебра, можно считать, что все множества $A_{k}$ попарно не пересекаются и все $a_{k}$ отличны от нуля. Тогда $\bigcup\left(A_{k} \mid k \in K\right)=\operatorname{coz} v \subset C$. Следовательно, каждое множество $A_{k}$ является предкомпактным. По лемме $6 \chi\left(A_{k}\right) \in X_{\varphi}$. Значит, $v \in X_{\varphi}$. Аналогичным образом $w \in X_{\varphi}$.

Возьмем произвольное $\delta>0$. По доказанному выше для $\varepsilon \equiv \delta /(3 \widehat{\varphi}(x))$ подберем соответствующие $v$ и $w$ из $X_{\varphi}$. Для них существуют $g^{\prime}, g^{\prime \prime} \in Y$ и $h^{\prime}, h^{\prime \prime} \in Z$ такие, что $h^{\prime} \leqslant v \leqslant g^{\prime}, h^{\prime \prime} \leqslant w \leqslant g^{\prime \prime}, \bar{\varphi} g^{\prime}-\underline{\varphi} h^{\prime}<\delta / 3$ и $\bar{\varphi} g^{\prime \prime}-\underline{\varphi} h^{\prime \prime}<\delta / 3$. Ясно, что $h^{\prime} \leqslant f \leqslant g^{\prime \prime}$. Кроме того,

$$
\begin{aligned}
\bar{\varphi} g^{\prime \prime}-\underline{\varphi} h^{\prime} & =\left(\bar{\varphi} g^{\prime \prime}-\underline{\varphi} h^{\prime \prime}\right)+\left(\underline{\varphi} h^{\prime \prime}-\bar{\varphi} g^{\prime}\right)+\left(\bar{\varphi} g^{\prime}-\underline{\varphi} h^{\prime}\right) \\
& <2 \delta / 3+\widehat{\varphi} h^{\prime \prime}-\widehat{\varphi} g^{\prime} \leqslant 2 \delta / 3+\widehat{\varphi} w-\widehat{\varphi} v \\
& =2 \delta / 3+\widehat{\varphi}(w-v) \leqslant 2 \delta / 3+\varepsilon \widehat{\varphi} x=\delta .
\end{aligned}
$$

Следовательно, $f \in X_{\varphi}$.

Рассмотрим линейный положительньй функционал $\widehat{\varphi}$ на множестве $S_{c}(T, \mathscr{G})$ и докажем, что на этом множестве он является тонким.

Лемма 7. Для любого $A \in \mathscr{A}_{c}(T, \mathscr{G})$ и любого $\varepsilon>0$ существует $K \in \mathscr{C}$ maкое, что $K \subset A$ u $\widehat{\varphi}(\chi(A \backslash K))<\varepsilon$. 
ДокАЗАТЕЛЬСТво. Пусть $D$-открытоепредкомпактное множество, $g \equiv \chi(D)$. Так как $g=\sup \left\{f \in C_{c}(T, \mathscr{G})_{+} \mid f \leqslant g\right\}$, то для любого $\varepsilon>0$ существует $f \in C_{c}(T, \mathscr{G})_{+}$такое, что $f \leqslant g$ и $\bar{\varphi} g-\varepsilon / 2<\varphi f$. Рассмотрим открытое множество $D^{\prime} \equiv \operatorname{coz} f$ и функцию $g^{\prime} \equiv \chi\left(D^{\prime}\right)$. Так как $\varphi f=\bar{\varphi} f \leqslant \bar{\varphi} g^{\prime}$, то $\bar{\varphi} g-\varepsilon / 2<\bar{\varphi} g^{\prime}$. По лемме $6 g, g^{\prime} \in X_{\varphi}$. Поэтому

$$
\widehat{\varphi} g-\widehat{\varphi} g^{\prime}=\widetilde{\varphi} g-\widetilde{\varphi} g^{\prime}=\bar{\varphi} g-\bar{\varphi} g^{\prime}<\varepsilon / 2 .
$$

Рассмотрим замкнутые множества $K_{n} \equiv\{t \in T \mid f(t) \geqslant 1 / n\} \subset D^{\prime}$ и функции $h_{n} \equiv \chi\left(K_{n}\right)$. Ясно, что $K_{n}$ компактны. Так как $\left(K_{n} \mid n \in \mathbb{N}\right) \uparrow D^{\prime}$, то $\left(h_{n} \mid n \in\right.$ $\mathbb{N}) \uparrow g^{\prime}$. В силу поточечной $\sigma$-непрерывности функционала $\widehat{\varphi}$ получаем $\left(\widehat{\varphi} h_{n} \mid n \in\right.$ $\mathbb{N}) \uparrow \widehat{\varphi} g^{\prime}$. Поэтому сушествует $n$ такое, что $\widehat{\varphi} g^{\prime}-\varepsilon / 2 \leqslant \widehat{\varphi} h_{n} \leqslant \widehat{\varphi} g^{\prime}$. В результате $g-h_{n}=\chi\left(D \backslash K_{n}\right)$ влечет

$$
\widehat{\varphi}\left(\chi\left(D \backslash K_{n}\right)\right)=\widehat{\varphi} g-\widehat{\varphi} h_{n} \leqslant \widehat{\varphi} g-\widehat{\varphi} g^{\prime}+\varepsilon / 2<\varepsilon .
$$

Пусть теперь $C$ - компактное множество. Для множества $D$ и $\varepsilon>0$ согласно установленному выше сушествует компактное множество $L$ такое, что $L \subset D$ и $\widehat{\varphi}(\chi(D \backslash L))<\varepsilon$. Рассмотрим компактное множество $K \equiv C \cap L \subset C \cap D$. Тогда получаем

$$
\widehat{\varphi}(\chi(C \cap D \backslash K))=\widehat{\varphi}(\chi(C \cap(D \backslash L))) \leqslant \widehat{\varphi}(\chi(D \backslash L))<\varepsilon .
$$

Пусть $A \in \mathscr{A}_{c}(T, \mathscr{G})$. По лемме $5 A=\bigcup\left(C_{i} \cap D_{i} \mid i=1, \ldots, n\right)$. Для $\varepsilon>0$ существуют компактные множества $K_{i}$ такие, что $K_{i} \subset C_{i} \cap D_{i}$ и $\widehat{\varphi}\left(\chi\left(C_{i} \cap D_{i} \backslash K_{i}\right)\right)<$ $\varepsilon / n$. Рассмотрим компактное множество $K \equiv \bigcup\left(K_{i} \mid i=1, \ldots, n\right)$. Так как

$$
A \backslash K=\bigcup\left(C_{i} \cap D_{i} \backslash K \mid i=1, \ldots, n\right) \subset \bigcup\left(C_{i} \cap D_{i} \backslash K_{i} \mid i=1, \ldots, n\right),
$$

то

$$
\begin{aligned}
\widehat{\varphi}(\chi(A \backslash K)) & \leqslant \widehat{\varphi}\left(\sum\left(\chi\left(C_{i} \cap D_{i} \backslash K_{i}\right) \mid i=1, \ldots, n\right)\right) \\
& =\sum\left(\widehat{\varphi}\left(\chi\left(C_{i} \cap D_{i} \backslash K_{i}\right)\right) \mid i=1, \ldots, n\right)<\varepsilon .
\end{aligned}
$$

TEOPEMA 2. Пусть $(T, \mathscr{G})$ - локально компактное пространство и $\varphi \in$ $\left(C_{c}(T, \mathscr{G})^{\sim}\right)_{+}$. Тогда функционал $\widehat{\varphi}$ на $S_{c}(T, \mathscr{G})$ является линейным положительныц тонким. Более того, он является единственным линейным положительным тонким продолжением функиионала $\varphi$ на $S_{c}(T, \mathscr{G})$.

ДоказАтельство. Пусть $\left(A_{n} \in \mathscr{A}_{c}(T, \mathscr{G}) \mid n \in \mathbb{N}\right) \downarrow 0$ и $\varepsilon>0$. По лемме 7 для $A_{n}$ существует компактное множество $L_{n}$ такое, что $L_{n} \subset A_{n}$ и $\widehat{\varphi}\left(\chi\left(A_{n} \backslash L_{n}\right)\right)<$ $\varepsilon / 2^{n+1}$. Рассмотрим компактные множества $K_{n} \equiv \bigcap\left(L_{i} \mid i=1, \ldots, n\right) \subset A_{n}$. Так как $A_{n} \backslash K_{n}=\bigcup\left(A_{n} \backslash L_{i} \mid i=1, \ldots, n\right) \subset \bigcup\left(A_{i} \backslash L_{i} \mid i=1, \ldots, n\right)$, то

$$
\begin{aligned}
\widehat{\varphi}\left(\chi\left(A_{n} \backslash K_{n}\right)\right) & \leqslant \widehat{\varphi}\left(\sum\left(\chi\left(A_{i} \backslash L_{i}\right) \mid i=1, \ldots, n\right)\right) \\
& =\sum\left(\widehat{\varphi}\left(\chi\left(A_{i} \backslash L_{i}\right)\right) \mid i=1, \ldots, n\right) \\
& <\varepsilon \sum\left(1 / 2^{i+1} \mid i=1, \ldots, n\right) \leqslant \varepsilon / 2 .
\end{aligned}
$$


Рассмотрим компактное множество $K \equiv \bigcap\left(K_{n} \mid n \in \mathbb{N}\right)$ и функции $h \equiv \chi(K)$ и $h_{n} \equiv \chi\left(K_{n}\right)$. Так как $\left(K_{n} \mid n \in \mathbb{N}\right) \downarrow K$, то $\left(h_{n} \mid n \in \mathbb{N}\right) \downarrow h$. В силу поточечной $\sigma$-непрерывности функционала $\widehat{\varphi}$ получаем $\left(\widehat{\varphi} h_{n} \mid n \in \mathbb{N}\right) \downarrow \widehat{\varphi} h$. Следовательно, сушествует $n$ такое, что $\widehat{\varphi} h+\varepsilon / 2>\widehat{\varphi} h_{n}$. В результате

$$
\widehat{\varphi} \chi\left(A_{n} \backslash K\right)=\widehat{\varphi} \chi\left(A_{n}\right)-\widehat{\varphi} h<\widehat{\varphi} \chi\left(A_{n}\right)-\widehat{\varphi} h_{n}+\varepsilon / 2=\widehat{\varphi} \chi\left(A_{n} \backslash K_{n}\right)+\varepsilon / 2 \leqslant \varepsilon .
$$

Значит, функционал $\widehat{\varphi}$ является положительным внутренне компактным.

Далее, для любой точки $t \in T$ сушествует предкомпактная открытая окрестность $D$. Рассмотрим функцию $g \equiv \chi(D) \in X_{\varphi}$. Если $f \in X_{\varphi}$ и $|f| \leqslant g$, то $-\widehat{\varphi} g=\widehat{\varphi}(-g) \leqslant \widehat{\varphi} f \leqslant \widehat{\varphi} g$ влечет $|\widehat{\varphi} f| \leqslant \widehat{\varphi} g<\infty$. Значит, $\widehat{\varphi}$ является локально ограниченньгм.

Наконец, свойство поточечной $\sigma$-непрерывности следует из теоремы 1 . Таким образом, функционал $\widehat{\varphi}$ является тонким.

Пусть теперь $\psi$ является линейным положительным тонким функционалом на $S_{c}$, продолжаюшим функционал $\varphi$. Докажем, что $\psi=\widehat{\varphi}$.

Сначала докажем, что $\psi \chi(D)=\widehat{\varphi} \chi(D)$ для любого предкомпактного открытого множества $D$. Так как функционал $\psi$ является положительным внутренне компактным, то для любого $\varepsilon>0$ существует компактное множество $C \subset D$ такое, что $\psi \chi(D \backslash C)<\varepsilon$. Обозначим $\chi(D)$ и $\chi(C)$ через $g$ и $h$ соответственно. Следовательно, $\psi g<\psi h+\varepsilon$. Рассмотрим замкнутое множество $F \equiv T \backslash D$. Так как пространство $T$ тихоновское и множество $C$ компактное, то существует непрерьвная функция $f$ на $T$ такая, что $0 \leqslant f \leqslant \mathbf{1}, f(t)=0$ для любого $t \in F$ и $f(t)=1$ для любого $t \in C$. Ясно, что $h \leqslant f \leqslant g$. Поэтому $\psi g<\psi h+\varepsilon \leqslant \psi f+\varepsilon=\varphi f+\varepsilon$. Значит,

$$
\psi g=\sup \left\{\varphi f \mid f \in C_{c} \wedge f \leqslant g\right\} \equiv \bar{\varphi} g=\widehat{\varphi} g .
$$

Пусть теперь $K$ - произвольное компактное множество. Тогда существует предкомпактное открытое множество $D \supset K$. Рассмотрим предкомпактное отрытое множество $E \equiv D \backslash K$. Так как $\chi(K)=\chi(D)-\chi(E)$, то $\psi \chi(K)=\psi \chi(D)-$ $\psi \chi(E)=\widehat{\varphi} \chi(D)-\widehat{\varphi} \chi(E)=\widehat{\varphi} \chi(K)$.

Пусть теперь $A \in \mathscr{A}_{c}$. Так же, как и вьше, для $\varepsilon>0$ существует компактное множество $K \subset A$ такое, что $\psi \chi(A \backslash K)<\varepsilon / 2$. Аналогично, существует компактное множество $L \subset A$ такое, что $\widehat{\varphi} \chi(A \backslash L)<\varepsilon / 2$. Рассмотрим компактное множество $C \equiv K \cup L \subset A$. Так как $\psi$ и $\widehat{\varphi}$ возрастают, то $\psi \chi(A \backslash C)<\varepsilon / 2$ и $\widehat{\varphi} \chi(A \backslash C)<\varepsilon / 2$. Следовательно,

$$
|\psi \chi(A)-\widehat{\varphi} \chi(A)| \leqslant|\psi \chi(A)-\psi \chi(C)|+|\widehat{\varphi} \chi(C)-\widehat{\varphi} \chi(A)|<\varepsilon .
$$

Так как $\varepsilon$ произвольно, то $\psi \chi(A)=\widehat{\varphi} \chi(A)$.

Наконец, пусть $f \in S_{c}$. Возьмем произвольное $\delta>0$ и $\varepsilon \equiv \delta / \psi(x)$, где $x-$ функция из доказательства предложения 2. Для $\varepsilon$ рассмотрим функции $v$ и $w$ из доказательства предложения 2. Тогда $v=\sum\left(a_{k} \chi\left(A_{k}\right) \mid k \in K\right)$ для некоторой конечной коллекции $\left(A_{k} \in \mathscr{A}_{c} \mid k \in K\right)$. Следовательно,

$$
\psi v=\sum\left(a_{k} \psi \chi\left(A_{k}\right) \mid k \in K\right)=\sum\left(a_{k} \widehat{\varphi} \chi\left(A_{k}\right) \mid k \in K\right)=\widehat{\varphi} v .
$$

Аналогично, $\psi w=\widehat{\varphi} w$. Так как $v \leqslant f \leqslant w$ и $0 \leqslant w-v \leqslant \varepsilon x$, то

$$
\begin{aligned}
& \psi f \leqslant \psi w \leqslant \psi v+\varepsilon \psi x=\widehat{\varphi} v+\delta \leqslant \widehat{\varphi} f+\delta \\
& \widehat{\varphi} f \leqslant \widehat{\varphi} w \leqslant \widehat{\varphi} v+\varepsilon \widehat{\varphi} x=\psi v+\delta \leqslant \psi f+\delta .
\end{aligned}
$$


Следовательно, $|\psi f-\widehat{\varphi} f| \leqslant \delta$. Так как $\delta$ произвольно, то $\psi f=\widehat{\varphi} f$. Теорема доказана.

По этой теореме можно корректно определить отображение $P_{0}$ из $\left(C_{c}(T, \mathscr{G})^{\sim}\right)_{+}$ в $\left(S_{c}(T, \mathscr{G})^{\Delta}\right)_{+}$, полагая $P_{0} \varphi \equiv \widehat{\varphi}$.

СЛЕДСТВИЕ 1. Пусть $(T, \mathscr{G})$ - локально компактное пространство. Тогда отобрахсение $P_{0}$ из $\left(C_{c}(T, \mathscr{G})^{\sim}\right)_{+}$в $\left(S_{c}(T, \mathscr{G})^{\Delta}\right)_{+}$является биективным.

ДокаЗАТЕЛЬСТво. Если $\varphi^{\prime} \neq \varphi^{\prime \prime}$, то, очевидно, $\widehat{\varphi}^{\prime} \neq \hat{\varphi}^{\prime \prime}$. Значит, $P_{0}$ инъективно. Если $\psi \in\left(S_{c}^{\Delta}\right)_{+}$, то функционал $\varphi \equiv \psi \mid C_{c}$ является линейным и положительным. Рассмотрим его продолжение $\widehat{\varphi}$. По теореме $2 \psi=\widehat{\varphi}$. Значит, $P_{0}$ биективно.

Теперь можно доказать основную теорему этого пункта.

Теорема 3 (о следствии). Радоновское представление для локально компактного пространства следует из общего радоновского представления для произвольного хаусдорфова пространства.

ДокАЗАТЕЛЬСТво. Пусть $(T, \mathscr{G})$ - локально компактное пространство. По теореме 2 сушествует биекция $P_{0}$ из $\left(C_{c} \sim\right)_{+}$на $\left(S_{c}^{\Delta}\right)_{+}$. По теореме 1 из $[39, \S 2$, п. 5] об общем радоновском представлении сушествует биекция $V$ из $\left(S_{c}^{\Delta}\right)_{+}$на $\left(\mathfrak{R M}^{\text {wo }}\right)_{0}$. Биекция $V \circ P_{0}$ дает необходимое радоновское представление.

Следствие 1 можно значительно уточнить.

Теорема 4. Отображение $P_{0}$ имеет единственное расиирение до такого изоморфизма $P$ из решеточного векторного пространства $C_{c}(T, \mathscr{G})^{\sim}$ на решеточное векторное пространство $S_{c}(T, \mathscr{G})^{\Delta}$, что $P \varphi=P_{0}\left(\varphi_{+}\right)-P_{0}\left(-\varphi_{-}\right)$ для каждого $\varphi \in C_{c}(T, \mathscr{G})^{\sim}$.

ДокаЗАТЕЛЬСтво. Проверим сначала, что $P_{0}(a \varphi)=a P_{0} \varphi$ и $P_{0}\left(\varphi^{\prime}+\varphi^{\prime \prime}\right)=$ $P_{0} \varphi^{\prime}+P_{0} \varphi^{\prime \prime}$ для любых $a \in \mathbb{R}_{+}$и $\varphi^{\prime}, \varphi^{\prime \prime} \in\left(C_{c} \sim\right)_{+}$.

Можно считать, что $a>0$. Обозначим $а \varphi$ через $\psi$. Возьмем любые $g \in Y$ и $\varepsilon>0$. Рассмотрим множество $L_{g} \equiv\left\{f \in C_{c} \mid f \leqslant g\right\}$. Тогда из $\bar{\varphi} g=\sup (\varphi f \mid$ $\left.f \in L_{g}\right)$ и $\bar{\psi} g=\sup \left(\psi f \mid f \in L_{g}\right)$ следует, что сушествуют $u, v \in L_{g}$ такие, что $\bar{\varphi} g-\varepsilon /(2 a)<\varphi u$ и $\bar{\psi} g-\varepsilon / 2<\psi v$. Рассмотрим функцию $w \equiv u \vee v \in L_{g}$. Тогда

$$
|(a \bar{\varphi}) g-\bar{\psi} g| \leqslant|a \bar{\varphi} g-a \varphi w|+|\psi w-\bar{\psi} g|<a|\bar{\varphi} g-\varphi w|+\varepsilon / 2<\varepsilon .
$$

Из произвольности $\varepsilon$ следует, что $(a \bar{\varphi}) g=\bar{\psi} g$.

Аналогичным образом доказывается, что $(a \underline{\varphi}) h=\underline{\psi} h$ для любого $h \in Z$.

Пусть теперь $f \in S_{c}$ и $\varepsilon>0$. Рассмотрим множество $Z_{f} \equiv\{h \in Z \mid h \leqslant f\}$. Тогда из $\widehat{\varphi} f=\underline{\varphi f}=\sup \left(\underline{\varphi} h \mid h \in Z_{f}\right)$ и $\widehat{\psi} f=\sup \left(\underline{\psi} h \mid h \in Z_{f}\right)$ так же, как и выше, следует, что $(a \widehat{\varphi}) f=\widehat{\psi} f$.

Обозначим $\varphi^{\prime}+\varphi^{\prime \prime}$ через $\psi$. Пусть $g \in Y$ и $\varepsilon>0$. Тогда из $\bar{\varphi}^{\prime} g=\sup \left(\varphi^{\prime} f \mid f \in\right.$ $\left.L_{g}\right), \bar{\varphi}^{\prime \prime} g=\sup \left(\varphi^{\prime \prime} f \mid f \in L_{g}\right)$ и $\bar{\psi} g=\sup \left(\psi f \mid f \in L_{g}\right)$ следует, что существуют $u, v, w \in L_{g}$ такие, что $\bar{\varphi}^{\prime} g-\varepsilon / 3<\varphi^{\prime} u, \bar{\varphi}^{\prime \prime} g-\varepsilon / 3<\varphi^{\prime \prime} v$ и $\bar{\psi} g-\varepsilon / 3<\psi w$. Рассмотрим функцию $x \equiv u \vee v \vee w \in L_{g}$. Тогда

$$
\left|\left(\bar{\varphi}^{\prime}+\bar{\varphi}^{\prime \prime}\right) g-\bar{\psi} g\right| \leqslant\left|\bar{\varphi}^{\prime} g-\varphi^{\prime} x\right|+\left|\bar{\varphi}^{\prime \prime} g-\varphi^{\prime \prime} x\right|+|\psi x-\bar{\psi} g|<\varepsilon .
$$

Следовательно, $\left(\bar{\varphi}^{\prime}+\bar{\varphi}^{\prime \prime}\right) g=\bar{\psi} g$. 
Аналогичным образом доказывается, что $\left(\underline{\varphi}^{\prime}+\underline{\varphi}^{\prime \prime}\right) h=\underline{\psi} h$ для любого $h \in Z$.

Пусть теперь $f \in S_{c}$ и $\varepsilon>0$. Тогда $\widehat{\varphi}^{\prime} f=\underline{\varphi^{\prime} f}=\overline{\sup }\left(\underline{\varphi^{\prime}} h \mid h \in Z_{f}\right)$, $\widehat{\varphi}^{\prime \prime} f=\sup \left(\underline{\varphi}^{\prime \prime} h \mid h \in Z_{f}\right)$ и $\widehat{\psi} f=\sup \left(\underline{\psi} h \mid h \in Z_{f}\right)$. Так же, как и выше, отсюда следует, что $\left(\widehat{\varphi}^{\prime}+\widehat{\varphi}^{\prime \prime}\right) f=\widehat{\psi} f$.

Проверим теперь, что отображение $P_{0}$ изотонно и сохраняет любые порядковые гранищы. Пусть $\varphi \leqslant \psi$. Возьмем любое $g \in Y$ и рассмотрим множество $L_{g} \equiv\{f \in$ $\left.C_{c} \mid f \leqslant g\right\}$. Тогда из $\bar{\varphi} g=\sup \left(\varphi f \mid f \in L_{g}\right)$ и $\bar{\psi} g=\sup \left(\psi f \mid f \in L_{g}\right)$ следует, что $\bar{\varphi} g \leqslant \bar{\psi} g$.

Возьмем теперь любое $f \in S_{c}$ и рассмотрим множество $Y^{f} \equiv\{g \in Y \mid g \geqslant f\}$. Тогда из $\widehat{\varphi} f=\widetilde{\varphi} f=\inf \left(\bar{\varphi} g \mid g \in Y^{f}\right)$ и $\widehat{\psi} f=\inf \left(\bar{\psi} g \mid g \in Y^{f}\right)$ следует, что $\widehat{\varphi} f \leqslant \widehat{\psi} f$. Таким образом, $P_{0}$ является монотонным. Из биективности $P_{0}$ теперь следует, что $P_{0}$ является изотонным. Следовательно, $P_{0}$ сохраняет любые порядковые границы.

В силу предложения 3.6.1 из [24] отображение $P_{0}$ имеет единственное расширение до инъективного линейного оператора $P$ из $A \equiv C_{c} \sim{ }^{\sim} B \equiv S_{c}^{\Delta}$ такого, что $P \varphi=P_{0}\left(\varphi_{+}\right)-P_{0}\left(-\varphi_{-}\right)$.

Так как $P_{0}$ сохраняет любые порядковые границы, то $\varphi_{1} \wedge \varphi_{2}=0$ влечет $P \varphi_{1} \wedge$ $P \varphi_{2}=P_{0} \varphi_{1} \wedge P_{0} \varphi_{2}=P_{0}\left(\varphi_{1} \wedge \varphi_{2}\right)=0$. В силу $[12$, п. $14, \mathrm{E}(\mathrm{b})] P$ является решеточным линейным оператором.

Если $\xi \in B$, то $\xi=\xi_{+}+\xi_{-}$. Согласно следствию $1 \xi_{+}=P_{0} \varphi^{\prime}$ и $-\xi_{-}=P_{0} \varphi^{\prime \prime}$ для некоторых $\varphi^{\prime}, \varphi^{\prime \prime} \in A_{+}$. Рассмотрим функционал $\varphi \equiv \varphi^{\prime}-\varphi^{\prime \prime} \in A$. Тогда $\xi=$ $P_{0} \varphi^{\prime}-P_{0} \varphi^{\prime \prime}=P \varphi^{\prime}-P \varphi^{\prime \prime}=P \varphi$. Это означает, что $P$ является сюръективным. Таким образом, $P$ - биективный решеточный линейный оператор. Поэтому $P$ необходимый изоморфизм.

7. Теорема о следствии для тихоновского пространства. В этом пункте покажем, что из общего ограниченного радоновского представления следует ограниченное радоновское представление для тихоновского пространства.

Пусть $(T, \mathscr{G})$ - тихоновское пространство и $\varphi$ - линейный положительный узкий вешественный функционал на решеточном векторном пространстве $C_{b}(T, \mathscr{G})$ всех ограниченных непрерывных вещественнозначных функций на $T$, т.е. $\varphi \in$ $\left(C_{b}(T, \mathscr{G})^{\pi}\right)_{+}$.

Покажем, что функционал $\varphi$ продолжается до линейного положительного тонкого функционала $\widehat{\varphi}$ на решеточном векторном пространстве $S(T, \mathscr{G})$ всех метаполунепрерывных функций на $T$.

Так же, как и в предыдущем пункте, следующая лемма является ключевой для дальнейших рассуждений. Поэтому приведем ее с доказательством, хотя она является хорошо известной.

ЛЕмма 1. Функционал $\varphi$ на $C_{b}(T, \mathscr{G})$ является поточечно непрерывныл.

ДокАЗАтЕльство. Пусть $\left(f_{m} \in C_{b} \mid m \in M\right) \downarrow 0$ в $F(T)$. Если $\varphi f_{m}=0$ для всех $m \in M$, то все доказано. Поэтому предположим, что существует $m_{0}$ такое, что $\varphi f_{m_{0}}>0$. Тогда $\left\|f_{m_{0}}\right\|_{u}>0$ и $\varphi \mathbf{1}>0$. Рассмотрим $M_{0} \equiv\left\{m \in M \mid m \geqslant m_{0}\right\}$. Ясно, что $\left(f_{m} \mid m \in M_{0}\right) \downarrow 0$. Рассмотрим $g_{m} \equiv f_{m} /\left\|f_{m_{0}}\right\|_{u}$. Тогда $0 \leqslant g_{m} \leqslant$ $g_{m_{0}} \leqslant \mathbf{1}$ для любого $m \in M_{0}$ и $\left(g_{m} \mid m \in M_{0}\right) \downarrow 0$.

Пусть для $\varepsilon>0$ компактное множество $K$ таково, что $f \in C_{b}$ и $|f| \leqslant \chi(T \backslash K)$ влечет $|\varphi f|<\varepsilon / 2$. Возьмем $\delta \equiv \varepsilon /(2 \varphi \mathbf{1})$. Рассмотрим открытые множества $G_{m} \equiv\left\{t \in T \mid g_{m}(t)<\delta\right\}$. Так как $\left(g_{m} \mid m \in M_{0}\right) \downarrow 0$, то $\left(G_{m} \mid m \in\right.$ 
$\left.M_{0}\right)=T$. Выделим конечное покрытие $\left(G_{n} \mid n \in N \subset M_{0}\right)$ множества $K$. Для $N$ сушествует $m$ такое, что $m \geqslant n$ для всех $n \in N$. Если $t \in K$, то $t \in G_{n}$. Поэтому $g_{m}(t) \leqslant g_{n}(t)<\delta$. Рассмотрим непрерьвную функцию $h_{m} \equiv\left(g_{m}-\delta \mathbf{1}\right) \vee 0 \leqslant \mathbf{1}$. Так как $0 \leqslant h_{m} \leqslant \chi(T \backslash K)$, то $\varphi g_{m}-\delta \varphi \mathbf{1} \leqslant \varphi h_{m}<\varepsilon / 2$. Следовательно, $\varphi g_{m}<\varepsilon / 2+\varepsilon / 2=\varepsilon$. Значит, $\left(\varphi g_{m} \mid m \in M_{0}\right) \downarrow 0$. Отсюда $\left(\varphi f_{m} \mid m \in M_{0}\right) \downarrow 0$ и, окончательно, $\left(\varphi f_{m} \mid m \in M\right) \downarrow 0$.

Рассмотрим множество $S\left(T, C_{b}(T, \mathscr{G})\right)$ всех ограниченных функций $f \in F_{b}(T)$ таких, что $f=\sup \left(f_{m} \in C_{b}(T, \mathscr{G}) \mid m \in M\right)$ в $F(T)$ для некоторой сети $\left(f_{m} \in C_{b}(T, \mathscr{G}) \mid m \in M\right)$, возрастаюшей вверх. Аналогичным образом рассмотрим множество $I\left(T, C_{b}(T, \mathscr{G})\right)$ всех функций $f \in F_{b}(T)$ таких, что $f=\inf \left(f_{m} \in\right.$ $\left.C_{b}(T, \mathscr{G}) \mid m \in M\right)$ в $F(T)$ для некоторой сети $\left(f_{m} \in C_{b}(T, \mathscr{G}) \mid m \in M\right)$, убывающей вниз. Ясно, что $C_{b}(T, \mathscr{G})=S\left(T, C_{b}(T, \mathscr{G})\right) \cap I\left(T, C_{b}(T, \mathscr{G})\right)$.

Далее вместо $S\left(T, C_{b}(T, \mathscr{G})\right)$ и $I\left(T, C_{b}(T, \mathscr{G})\right)$ временно будем писать просто $Y$ и $Z$ соответственно.

Определим на $Y$ вешественный функционал $\bar{\varphi}$, полагая $\bar{\varphi} g \equiv \sup \{\varphi f \mid f \in$ $\left.C_{b}(T, \mathscr{G}) \wedge f \leqslant g\right\}$ для каждого $g \in Y$. Аналогичным образом определим на $Z$ вещественный функционал $\underline{\varphi}$, полагая $\underline{\varphi} h \equiv \inf \left\{\varphi f \mid f \in C_{b}(T, \mathscr{G}) \wedge f \geqslant h\right\}$ для каждого $h \in Z$. Ясно, что $\bar{\varphi}$ и $\underline{\varphi}$ являются продолжениями $\varphi$.

Теперь определим на $F_{b}(T)$ вешественные функционалы $\widetilde{\varphi}$ и $\varphi$, полагая $\widetilde{\varphi} f \equiv$ $\inf \{\bar{\varphi} g \mid g \in Y \wedge g \geqslant f\}$ и $\underline{\varphi} f \equiv \sup \{\underline{\varphi} h \mid h \in Z \wedge h \leqslant f\}$ для каждой функции $f \in F_{b}(T)$. Ясно, что $\widetilde{\varphi}$ является продолжением $\bar{\varphi}$, а $\varphi$ является продолжением $\underline{\varphi}$.

Рассмотрим в $F_{b}(T)$ подмножество $X_{\varphi} \equiv D\left(T, \widetilde{C}_{b}(T, \mathscr{G}), \varphi\right) \equiv\left\{f \in F_{b}(T)\right.$ $\widetilde{\varphi} f=\varphi f\}$. Определим вешественньй функционал $\widehat{\varphi}$ на $X_{\varphi}$, положив $\widehat{\varphi} f \equiv \widetilde{\varphi} f=$ $\varphi f$.

Для $Y, Z, X_{\varphi}, \bar{\varphi}, \underline{\varphi}, \widetilde{\varphi}, \underline{\varphi}$ и $\widehat{\varphi}$ справедливы леммы $2-4$, предложение 1 и теорема 1 из предыдущего пункта.

Докажем теперь, что $S(T, \mathscr{G}) \subset D\left(T, C_{b}(T, \mathscr{G}), \varphi\right)$.

Лемма 2. Пусть $A \in \mathscr{A}(T, \mathscr{G})$. Тогда $\chi(A) \in X_{\varphi}$.

ДоказАТЕльСтво. Если $G$ - открытое множество, то $g \equiv \chi(G) \in Y \subset X_{\varphi}$. Если $F$ - замкнутое множество, то $h \equiv \chi(F) \in Z \subset X_{\varphi}$. Так как $X_{\varphi}$ - решетка, то $\chi(F \cap G)=g \wedge h \in X_{\varphi}$.

Так как $A=\bigcup\left(F_{i} \cap G_{i} \mid i \in I\right)$ для некоторых конечных коллекций $\left(F_{i} \in \mathscr{F} \mid\right.$ $i \in I)$ и $\left(G_{i} \in \mathscr{G} \mid i \in I\right)$, то $\chi(A)=\sup \left(\chi\left(F_{i} \cap G_{i}\right) \mid i \in I\right) \in X_{\varphi}$.

ПРЕДЛОЖЕНИЕ 1. $S(T, \mathscr{G}) \subset D\left(T, C_{b}(T, \mathscr{G}), \varphi\right)$.

ДокаЗАТельСтво. Пусть $f \in S$. Возьмем любое $\varepsilon>0$. При доказательстве теоремы 1 из $[39, \S 2$, п. 4] было установлено, что для $\varepsilon$ существует ступенчатая функция $u \in \operatorname{St}(T, \mathscr{A}(T, \mathscr{G}))$ такая, что $|f(t)-u(t)|<\varepsilon / 4$ для всех $t \in T$. Возьмем $v \equiv u-(\varepsilon / 4) \mathbf{1}$ и $w \equiv u+(\varepsilon / 4) \mathbf{1}$. Тогда $v(t) \leqslant f(t) \leqslant w(t), v(t)>f(t)-\varepsilon / 2$ и $w(t)<f(t)+\varepsilon / 2$ для всех $t \in T$. Следовательно, $0 \leqslant w-v \leqslant \varepsilon x$, где $x \equiv \mathbf{1}$.

Так как $v \in \operatorname{St}(T, \mathscr{A}(T, \mathscr{G}))$, то $v=\sum\left(a_{k} \chi\left(A_{k}\right) \mid k \in K\right)$ для некоторых конечных коллекций $\left(a_{k} \in \mathbb{R} \mid k \in K\right)$ и $\left(A_{k} \in \mathscr{A}(T, \mathscr{G}) \mid k \in K\right)$. В силу леммы 2 $v \in X_{\varphi}$. Аналогично, $w \in X_{\varphi}$.

Далее рассуждением, полностью повторяюшим соответствующее рассуждение из доказательства предложения 2 п. 6 , убеждаемся, что $f \in X_{\varphi}$.

Рассмотрим теперь линейный положительный функционал $\widehat{\varphi}$ на $S(T, \mathscr{G})$ и докажем, что на этом множестве он является тонким. 
Лемма 3. Для любого $A \in \mathscr{A}(T, \mathscr{G})$ и любого $\varepsilon>0$ существует $K \in \mathscr{C}$ maкoe, что $K \subset A$ u $\widehat{\varphi}(\chi(A \backslash K))<\varepsilon$.

ДокАЗАТЕЛЬСТВо. Пусть $G$ - открытое множество и $g \equiv \chi(G)$. Возьмем любое $\varepsilon>0$. Рассмотрим множество $B \equiv\left\{f \in C_{b} \mid 0 \leqslant f \leqslant g\right\}$. Так как $\bar{\varphi} g=\sup (\varphi f \mid f \in B)$, то сушествует $f \in B$ такое, что $\bar{\varphi} g-\varepsilon / 4<\varphi f$. Рассмотрим функции $f_{n} \equiv(f-(1 / n) \mathbf{1}) \vee 0 \in B$. Так как $\left(f_{n} \mid n \in \mathbb{N}\right) \uparrow f$, то по лемме 1 $\left(\varphi f_{n} \mid n \in \mathbb{N}\right) \uparrow \varphi f$. Поэтому сушествует $m$ такое, что $\varphi f-\varepsilon / 4<\varphi f_{m}$. В результате $\bar{\varphi} g-\varepsilon / 2<\varphi f_{m}$. Если $t \in \operatorname{coz} f_{m}$, то $f(t)-1 / m>0$. Следовательно, $\operatorname{coz} f_{m} \subset$ $\{t \in T \mid f(t)>1 / m\}$ влечет $C \equiv \operatorname{clcoz} f_{m} \subset\{t \in T \mid f(t) \geqslant 1 / m\} \subset \operatorname{coz} f \subset G$. Для замкнутого множества $C$ мы получаем неравенство $\widehat{\varphi} g-\varepsilon / 2<\widehat{\varphi} f_{m} \leqslant \widehat{\varphi} \chi(C)$, откуда $\widehat{\varphi} \chi(G \backslash C)=\widehat{\varphi} g-\widehat{\varphi} \chi(C)<\varepsilon / 2$.

Так как функционал $\varphi$ является узким, то сушествует компактное множество $D$ такое, что $f \in C_{b}$ и $|f| \leqslant \chi(T \backslash D)$ влечет $|\varphi f|<\varepsilon / 2$. Это означает, что $\widehat{\varphi} \chi(T \backslash$ $D)=\bar{\varphi} \chi(T \backslash D) \leqslant \varepsilon / 2$.

Рассмотрим компактное множество $L \equiv C \cap D \subset G$. Так как $G \backslash L \subset(G \backslash C) \cup(T \backslash$ $D)$, то $\chi(G \backslash L) \leqslant \chi(G \backslash C)+\chi(T \backslash D)$ влечет $\widehat{\varphi} \chi(G \backslash L) \leqslant \widehat{\varphi} \chi(G \backslash C)+\widehat{\varphi} \chi(T \backslash D)<\varepsilon$.

Пусть $F$ - замкнутое множество. Согласно установленному выше, для $\varepsilon>0$ и множества $G$ существует компактное множество $L \subset G$ такое, что $\widehat{\varphi}(\chi(G \backslash L))<\varepsilon$. Рассмотрим компактное множество $K \equiv F \cap L \subset F \cap G$. Тогда $F \cap G \backslash K=F \cap$ $G \backslash F \cap L=F \cap(G \backslash L) \subset G \backslash L$ влечет $\chi(F \cap G \backslash K) \leqslant \chi(G \backslash L)$. Следовательно, $\widehat{\varphi}(\chi(F \cap G \backslash K)) \leqslant \widehat{\varphi}(\chi(G \backslash L))<\varepsilon$.

Пусть теперь $A \in \mathscr{A}(T, \mathscr{G})$. Тогда $A=\bigcup\left(F_{i} \cap G_{i} \mid i=1, \ldots, n\right)$. Для $\varepsilon>0$ сушествуют компактные множества $K_{i}$ такие, что $K_{i} \subset F_{i} \cap G_{i}$ и $\widehat{\varphi}\left(\chi\left(F_{i} \cap G_{i} \backslash\right.\right.$ $\left.\left.K_{i}\right)\right)<\varepsilon / n$. Рассмотрим компактное множество $K \equiv \bigcup\left(K_{i} \mid i=1, \ldots, n\right)$. Так как $A \backslash K \subset \bigcup\left(F_{i} \cap G_{i} \backslash K_{i} \mid i=1, \ldots, n\right)$, то $\widehat{\varphi}(\chi(A \backslash K)) \leqslant \widehat{\varphi}\left(\sum\left(\chi\left(F_{i} \cap G_{i} \backslash\right.\right.\right.$ $\left.\left.\left.K_{i}\right) \mid i=1, \ldots, n\right)\right)=\sum\left(\widehat{\varphi}\left(\chi\left(F_{i} \cap G_{i} \backslash K_{i}\right)\right) \mid i=\mathbf{1}, \ldots, n\right)<\varepsilon$.

TеОРема 1. Пусть $(T, \mathscr{G})$ - тихоновское пространство и $\varphi \in\left(C_{b}(T, \mathscr{G})^{\pi}\right)_{+}$. Тогда функционал $\widehat{\varphi}$ на $S(T, \mathscr{G})$ является линейным положительным тонким. Более того, он является единственным линейным положительным. тонким продолжением функиионала $\varphi$ на $S(T, \mathscr{G})$.

ДокАЗАТЕЛЬСТво. Тонкость функционала $\widehat{\varphi}$ устанавливается точно так же, как и при доказательстве теоремы 2 из п. 6.

Пусть теперь $\psi$ является линейным положительным тонким функционалом на $S$, продолжающим функционал $\varphi$. Докажем, что $\psi=\widehat{\varphi}$.

Пусть $G$ - произвольное открытое множество. Так как $\psi$ является положительным внутренне компактным, то для любого $\varepsilon>0$ существует компактное множество $C \subset G$ такое, что $\psi \chi(G \backslash C)<\varepsilon$. Обозначим $\chi(G)$ и $\chi(C)$ через $g$ и $h$ соответственно. Далее точно так же, как и при доказательстве теоремы 2 из п. 6 , устанавливается, что $\psi g=\widehat{\varphi} g$.

Пусть теперь $F$ - произвольное замкнутое множество. Рассмотрим открытое множество $G \equiv T \backslash F$. Так как $\chi(F)=\mathbf{1}-\chi(G)$, то $\psi \chi(F)=\psi \mathbf{1}-\psi \chi(G)=\widehat{\varphi} \mathbf{1}-$ $\widehat{\varphi} \chi(G)=\widehat{\varphi} \chi(F)$.

Пусть теперь $A \in \mathscr{A}$. Так же, как и при доказательстве теоремы 2 из п. 6 , устанавливается, что $\psi \chi(A)=\widehat{\varphi} \chi(A)$.

Наконец, пусть $f \in S$. Возьмем произвольное $\delta>0$ и $\varepsilon \equiv \delta / \psi(x)$, где $x \equiv \mathbf{1}$. Для $\varepsilon$ возьмем функции $v$ и $w$ из доказательства предложения 1 . Тогда $v=\sum\left(a_{k} \chi\left(A_{k}\right) \mid\right.$ 
$k \in K)$ для некоторой конечной коллекции $\left(A_{k} \in \mathscr{A} \mid k \in K\right)$. Следовательно, $\psi v=\sum\left(a_{k} \psi \chi\left(A_{k}\right) \mid k \in K\right)=\sum\left(a_{k} \widehat{\varphi} \chi\left(A_{k}\right) \mid k \in K\right)=\widehat{\varphi} v$. Аналогично, $\psi w=\widehat{\varphi} w$. Далее точно так же, как и при доказательстве упомянутой теоремы 2 , устанавливается, что $\psi f=\widehat{\varphi} f$.

По этой теореме можно корректно определить отображение $Q_{0}$ из $\left(C_{b}(T, \mathscr{G})^{\pi}\right)_{+}$ в $\left(S(T, \mathscr{G})^{\Delta}\right)_{+}$, полагая $Q_{0} \varphi \equiv \widehat{\varphi}$.

СлеДСТВИЕ 1. Пусть $(T, \mathscr{G})$ - тихоновское пространство. Тогда отображение $Q_{0}$ из $\left(C_{b}(T, \mathscr{G})^{\pi}\right)_{+}$в $\left(S(T, \mathscr{G})^{\Delta}\right)_{+}$является биективным.

ДокАЗАТЕЛЬСТво. Если $\varphi^{\prime} \neq \varphi^{\prime \prime}$, то, очевидно, $\widehat{\varphi}^{\prime} \neq \widehat{\varphi}^{\prime \prime}$. Значит, $Q_{0}$ инъективно. Если $\psi \in\left(S_{c}^{\Delta}\right)_{+}$, то функционал $\varphi \equiv \psi \mid C_{b}$ является линейным и положительным. Пусть $\varepsilon>0$. Так как $\psi$ является положительным внутренне компактным, то для $T$ сушествует компактное множество $K$ такое, что $\psi \chi(T \backslash K)<\varepsilon$. Если $f \in C_{b}$ и $|f| \leqslant \chi(T \backslash K)$, то $|\varphi f|=|\psi f|<\varepsilon$. Значит, $\varphi$ является узким. Рассмотрим его продолжение $\widehat{\varphi}$. По теореме $1 \psi=\widehat{\varphi}$. Значит, $Q_{0}$ биективно.

ЛЕмма 4. Отображение $Q_{0}$ является положительно линейным в том смислле, ито $Q_{0}(a \varphi)=а Q_{0} \varphi$ и $Q_{0}\left(\varphi^{\prime}+\varphi^{\prime \prime}\right)=Q_{0} \varphi^{\prime}+Q_{0} \varphi^{\prime \prime}$ для любых $a \in \mathbb{R}_{+}$ $u \varphi^{\prime}, \varphi^{\prime \prime} \in\left(C_{b}(T, \mathscr{G})^{\pi}\right)_{+}$.

ДокАЗАТЕЛЬСтво. Пусть $a>0$. Обозначим $a \varphi$ через $\psi$. Возьмем любые $g \in Y$ и $\varepsilon>0$. Рассмотрим множество $L_{g} \equiv\left\{f \in C_{b} \mid f \leqslant g\right\}$. Тогда из $\bar{\varphi} g=\sup \left(\varphi f \mid f \in L_{g}\right)$ и $\bar{\psi} g=\sup \left(\psi f \mid f \in L_{g}\right)$ следует, что существуют $u, v \in L_{g}$ такие, что $\bar{\varphi} g-\varepsilon /(2 a)<\varphi u$ и $\bar{\psi} g-\varepsilon / 2<\psi v$. Рассмотрим функцию $w \equiv u \vee v \in L_{g}$. Тогда

$$
|(a \bar{\varphi}) g-\bar{\psi} g| \leqslant|a \bar{\varphi} g-a \varphi w|+|\psi w-\bar{\psi} g|<a|\bar{\varphi} g-\varphi w|+\varepsilon / 2<\varepsilon .
$$

Из произвольности $\varepsilon$ следует, что $(a \bar{\varphi}) g=\bar{\psi} g$.

Аналогично доказывается, что $(a \underline{\varphi}) h=\underline{\psi} h$ для любого $h \in Z$.

Пусть теперь $f \in S$ и $\varepsilon>0$. Рассмотрим множество $Z_{f} \equiv\{h \in Z \mid h \leqslant f\}$. Тогда из $\widehat{\varphi} f=\underline{\varphi} f=\sup \left(\underline{\varphi} h \mid h \in Z_{f}\right)$ и $\widehat{\psi} f=\sup \left(\underline{\psi} h \mid h \in Z_{f}\right)$ так же, как и вьше, следует, что $(a \widehat{\varphi}) f=\widehat{\psi} f$.

Обозначим $\varphi^{\prime}+\varphi^{\prime \prime}$ через $\psi$. Пусть $g \in Y$ и $\varepsilon>0$. Тогда из $\bar{\varphi}^{\prime} g=\sup \left(\varphi^{\prime} f \mid f \in\right.$ $\left.L_{g}\right), \bar{\varphi}^{\prime \prime} g=\sup \left(\varphi^{\prime \prime} f \mid f \in L_{g}\right)$ и $\bar{\psi} g=\sup \left(\psi f \mid f \in L_{g}\right)$ следует, что сушествуют $u, v, w \in L_{g}$ такие, что $\bar{\varphi}^{\prime} g-\varepsilon / 3<\varphi^{\prime} u, \bar{\varphi}^{\prime \prime} g-\varepsilon / 3<\varphi^{\prime \prime} v$ и $\bar{\psi} g-\varepsilon / 3<\psi w$. Рассмотрим функцию $x \equiv u \vee v \vee w \in L_{g}$. Тогда

$$
\left|\left(\bar{\varphi}^{\prime}+\bar{\varphi}^{\prime \prime}\right) g-\bar{\psi} g\right| \leqslant\left|\bar{\varphi}^{\prime} g-\varphi^{\prime} x\right|+\left|\bar{\varphi}^{\prime \prime} g-\varphi^{\prime \prime} x\right|+|\psi x-\bar{\psi} g|<\varepsilon
$$

Следовательно, $\left(\bar{\varphi}^{\prime}+\bar{\varphi}^{\prime \prime}\right) g=\bar{\psi} g$.

Аналогично доказывается, что $\left(\underline{\varphi}^{\prime}+\underline{\varphi}^{\prime \prime}\right) h=\underline{\psi} h$ для любого $h \in Z$.

Пусть теперь $f \in S$ и $\varepsilon>0$. Тогда $\overline{\hat{\varphi}}^{\prime} f=\underline{\varphi^{\prime}} f=\sup \left(\underline{\varphi^{\prime}} h \mid h \in Z_{f}\right), \widehat{\varphi}^{\prime \prime} f=$ $\sup \left(\underline{\varphi}^{\prime \prime} h \mid h \in Z_{f}\right)$ и $\widehat{\psi} f=\sup \left(\underline{\psi} h \mid h \in Z_{f}\right)$. Так же, как и выше, отсюда следует, что $\left(\widehat{\varphi}^{\prime}+\widehat{\varphi}^{\prime \prime}\right) f=\widehat{\psi} f$.

Лемма 5. Отображсение $Q_{0}$ изотонно и сохраняет любые порядковые границь. 
ДокаЗАТЕЛЬСТво. Пусть $\varphi \leqslant \psi$. Возьмем любое $g \in Y$ и рассмотрим множество $L_{g} \equiv\left\{f \in C_{b} \mid f \leqslant g\right\}$. Тогда из $\bar{\varphi} g=\sup \left(\varphi f \mid f \in L_{g}\right)$ и $\bar{\psi} g=\sup (\psi f \mid$ $f \in L_{g}$ ) следует, что $\bar{\varphi} g \leqslant \bar{\psi} g$.

Возьмем теперь любое $f \in S$ и рассмотрим множество $Y^{f} \equiv\{g \in Y \mid g \geqslant f\}$. Тогда из $\widehat{\varphi} f=\widetilde{\varphi} f=\inf \left(\bar{\varphi} g \mid g \in Y^{f}\right)$ и $\widehat{\psi} f=\inf \left(\bar{\psi} g \mid g \in Y^{f}\right)$ следует, что $\widehat{\varphi} f \leqslant \widehat{\psi} f$. Таким образом, $Q_{0}$ является монотонным. Так как $Q_{0}$ биективно, то $Q_{0}$ является изотонным. Следовательно, $Q_{0}$ сохраняет любые порядковые гранищы.

Лемма 6. Отображение $Q_{0}$ имеет единственное расиирение до такого изоморфизма $Q$ из решеточного векторного пространства $C_{b}(T, \mathscr{G})^{\pi}$ на решеточное векторное пространство $S\left(T, \mathscr{G}^{\Delta}{ }^{\Delta}\right.$, ито $Q \varphi=Q_{0}\left(\varphi_{+}\right)-Q_{0}\left(-\varphi_{-}\right)$ для каждого $\varphi \in C_{b}(T, \mathscr{G})^{\pi}$.

ДоКАЗАТЕЛЬСТВо. В силу предложения 3.6.1 из [24] отображение $Q_{0}$ имеет единственное расширение до инъективного линейного оператора $Q$ из $A \equiv C_{b}^{\pi}$ в $B \equiv S^{\Delta}$ такого, что $Q \varphi=Q_{0}\left(\varphi_{+}\right)-Q_{0}\left(-\varphi_{-}\right)$.

По лемме $5 Q_{0}$ сохраняет любые порядковые гранищы. Поэтому $\varphi_{1} \wedge \varphi_{2}=0$ влечет $Q \varphi_{1} \wedge Q \varphi_{2}=Q_{0} \varphi_{1} \wedge Q_{0} \varphi_{2}=Q_{0}\left(\varphi_{1} \wedge \varphi_{2}\right)=0$. В силу [12, п. 14, E(b)] $Q$ является решеточным линейным оператором.

Если $\xi \in B$, то $\xi=\xi_{+}-\left(-\xi_{-}\right)$. Согласно следствию $1 \xi_{+}=Q_{0} \varphi^{\prime}$ и $-\xi_{-}=Q_{0} \varphi^{\prime \prime}$ для некоторых $\varphi^{\prime}, \varphi^{\prime \prime} \in A_{+}$. Рассмотрим функционал $\varphi \equiv \varphi^{\prime}-\varphi^{\prime \prime} \in A$. Тогда $\xi=$ $Q_{0} \varphi^{\prime}-Q_{0} \varphi^{\prime \prime}=Q \varphi^{\prime}-Q \varphi^{\prime \prime}=Q \varphi$. Это означает, что $Q$ является сюръективным. Таким образом, $Q$ есть биективньй решеточный линейный оператор. Поэтому $Q$ есть необходимый изоморфизм.

Так как $C_{b}(T, \mathscr{G})^{\pi} \subset C_{b}(T, \mathscr{G})^{\sim} \subset\left(C_{b}(T, \mathscr{G}),\|\cdot\|_{u}\right)^{\prime}$ и $S(T, \mathscr{G})^{\Delta} \subset S(T, \mathscr{G})^{\sim} \subset$ $\left(S(T, \mathscr{G}),\|\cdot\|_{u}\right)^{\prime}$, то $C_{b}(T, \mathscr{G})^{\pi}=C_{b}(T, \mathscr{G})^{\pi}$ и $S(T, \mathscr{G})^{\otimes}=S(T, \mathscr{G})^{\Delta}$. Поэтому $Q$ является изоморфизмом из решеточного векторного пространства $C_{b}(T, \mathscr{G})^{(\pi)}$ на решеточное векторное пространство $S(T, \mathscr{G}) \otimes$.

Индуцируем на $C_{b}(T, \mathscr{G})^{\circledR}$ норму $\|\cdot\|$ из нормированного решеточного векторного пространства $\left(\left(C_{b}(T, \mathscr{G}),\|\cdot\|_{u}\right)^{\prime},\|\cdot\|\right)$, а на $S(T, \mathscr{G})^{\ominus}$ - норму $\|\cdot\|$ из нормированного решеточного векторного пространства $\left(\left(S(T, \mathscr{G}),\|\cdot\|_{u}\right)^{\prime},\|\cdot\|\right)$.

ТеОРема 2. Пусть $(T, \mathscr{G})$ - тихоновское пространство. Тогда отображение $Q$ является (изометрическим) изоморфизмом из нормированного решеточного векторного пространства $\left(C_{b}(T, \mathscr{G})^{\circledR},\|\cdot\|\right)$ на нормированное решеточное векторное пространств $(S(T, \mathscr{G}) \oplus,\|\cdot\|)$.

ДОКАЗАТЕЛЬСТВО. В силу леммы 6 нужно проверить только изометричность. Пусть $\varphi \in\left(C_{b}^{(\pi)}\right)_{+}$и $\widehat{\varphi}=Q \varphi$. Если $f \in C_{b}$, то $|\varphi f|=\|\widehat{\varphi} f\| \leqslant\|\widehat{\varphi}\|\|f\|$. Следовательно, $\|\varphi\| \leqslant\|\widehat{\varphi}\|$.

Пусть теперь $f \in S$. Тогда $|\widehat{\varphi} f| \leqslant \widehat{\varphi}|f|=\widetilde{\varphi}|f|=\sup \left\{\bar{\varphi} h\left|h \in Z_{+} \wedge h \leqslant\right| f \mid\right\}$. Так как $\bar{\varphi} h=\inf \left\{\varphi x \mid x \in C_{b} \wedge x \geqslant h\right\}$, то

$$
\begin{aligned}
\bar{\varphi} h & \leqslant \inf \left\{\varphi x \mid x \in C_{b} \wedge x \geqslant h\right\} \leqslant \inf \left\{\|\varphi\|\|x\|_{u} \mid x \in C_{b} \wedge x \geqslant h\right\} \\
& \leqslant\|\varphi\| \inf \left\{\|x\|_{u} \mid x \in C_{b} \wedge x \geqslant\|h\|_{u} \mathbf{1}\right\}=\|\varphi\|\|h\|_{u} .
\end{aligned}
$$

Следовательно,

$$
\begin{aligned}
|\widehat{\varphi} f| & \leqslant\|\varphi\| \sup \left\{\|h\|_{u}\left|h \in Z_{+} \wedge h \leqslant\right| f \mid\right\} \\
& \leqslant\|\varphi\| \sup \left\{\|h\|_{u} \mid h \in Z_{+} \wedge\|h\|_{u} \leqslant\|f\|_{u}\right\} \leqslant\|\varphi\|\|f\|_{u} .
\end{aligned}
$$


Значит, $\|\widehat{\varphi}\| \leqslant\|\varphi\|$. В результате $\|\varphi\|=\|Q \varphi\|$.

Пусть теперь $\varphi \in C_{b}^{\circledR \pi}$. Тогда $|\varphi|=\varphi \vee 0+(-\varphi) \vee 0$ в силу леммы 6 влечет $Q|\varphi|=|Q \varphi|$. Применяя равенство, доказанное вьше, получаем $\|\varphi\|=\||\varphi|\|=$ $\|Q|\varphi|\|=\||Q \varphi|\|=\|Q \varphi\|$.

Теперь можно доказать основную теорему этого пункта.

ТЕОРема 3 (о следствии). Ограниченное радоновское представление для тихоновского пространства следует из общего ограниченного радоновского представления для произвольного хаусдорфова пространства.

ДокАЗАТЕЛЬСТво. Пусть $(T, \mathscr{G})$ - тихоновское пространство. По теореме 2 существует изоморфизм $Q$ из $\left(C_{b}^{\mathbb{\pi}},\|\cdot\|\right)$ на $\left(S^{\otimes},\|\cdot\|\right)$. По теореме об общем ограниченном радоновском представлении существует изоморфизм $K$ из $(S \ominus,\|\cdot\|)$ на $\left(\mathfrak{R M}{ }_{b}^{w o},\|\cdot\|\right)$. Изоморфизм $K \circ Q$ дает необходимое радоновское представление.

\section{Список литературы}

1. Riesz F. Sur les opérations fonctionelles linéaires // C.R. Acad. Sci. Paris. 1909. V. 149. P. 974-977.

2. Radon J. Theorie und Anwendangen der absolut additiven Mengenfunktionen // S.-B. Akad. Wiss. Wien. 1913. V. 122. P. 1295-1438.

3. Hausdorff F. Grundzuge der Mengenlehre. Leipzig: Veit, 1914.

4. Saks S. Theory of the integral. N.Y.: Hafner, 1937.

5. Saks S. Integration in abstract metric spaces // Duke Math. J. 1938. V. 4. P. 408-411.

6. Kakutani S. Conerete representation of abstract (M) - spaces // Ann. Math. (2). 1941. V. 42. P. 994-1024.

7. Halmos P. R. Measure Theory. Princeton: Van Nostrand, 1950.

8. Hewitt E. Integration on locally compact spaces. I // Univ. of Washington Publ. in Math. 1952. V. 3. P. 71-75.

9. Edwads R. E. A theory of Radon measures on locally compact spaces // Acta Math. 1953. V. 89. P. $133-164$.

10. Bourbaki N. Intégration. Chap. I-VI. Paris: Hermann, 1952-1959.

11. Hewitt E., Stromberg K. Real and Abstract Analysis. Berlin: Springer-Verlag, 1965.

12. Fremlin D. H. Topological Riesz spaces and measure theory. Cambridge: The University Press, 1974.

13. Прохоров Ю. В. Сходимость случайных процессов и предельные теоремы теории вероятностей // Теор. вероятн. и ее применения. 1956. Т. 1. С. 177-238.

14. Topsoe F. Topology and measure // Lect. Notes Math. 1970. V. 133.

15. Pollard D., Topsoe F. A unified approach to Riesz type representation theorems // Studia Math. 1975. V. 54. P. 173-190.

16. Topsoe F. Further results on integral representations // Studia Math. 1976. V. 55. P. 239-245.

17. Topsoe F. Radon measures, some basic constructions // Lect. Notes Math. 1983. V. 1033. P. 303-311.

18. Anger B., Portenier C. Radon integrals. Boston-Berlin: Birkhäuser, 1992.

19. König H. The Danielle-Stone-Riesz representation theorem // Operator theory: advances and applications. 1995. V. 75. P. 191-222.

20. König H. Measure and integration. Berlin: Springer-Verlag, 1997.

21. Захаров B. K., Михалёв А. В. Проблема Радона для регулярных мер на произвольном хаусдорфовом пространстве // Фундам. и прикл. математика. 1997. Т. 3. № 3. С. 801-808.

22. Захаров B.K., Михалёв А.В. Интегральное представление для радоновских мер на произвольном хаусдорфовом пространстве // Фундам. и прикл. математика. 1997. Т. 3. № 4. C. $1135-1172$.

23. Захаров В.К., Михалёв А.В. Проблема интегрального представления для радоновских мер на произвольном хаусдорфовом пространстве // Докл. РАН. 1998. Т. 360. № 1. C. $13-15$. 
24. Semadeni Z. Banach spaces of continuous functions. Warszawa: Polish Sci. Publ., 1971.

25. Dinculianu N. Vector Measures. London-N.Y.: Oxford Univ. Press, 1967.

26. Александров А. Д. Additive functions in abstract spaces. I-III // Матем. сб. 1940. Т. 8. C. $303-348 ;$ 1941. T. 9. C. $563-628$; 1943. T. 13. C. 169-238.

27. Stone M. N. Notes on integration. I-IV // Proc. Nat. Acad. Sci. USA. 1948. V. 34. P. 336-342; 447-455; 483-490; 1949. V. 35. P. 50-58.

28. Schwartz L. Radon measures on arbitrary topological spaces and cylindrical measures. London-N.Y.: Oxford Univ. Press, 1973.

29. Jacobs K. Measure and integral. N.Y.: Academic Press, 1978.

30. Канторович Л. В., Акилов Г. П. Функциональный анализ. М.: Наука, 1977.

31. Захаров В. К. Функциональное представление равномерного пополнения максимального и счетно-плотного модулей частных модуля непрерывных функций // УМН. 1980. Т. 35. № 4. C. $187-188$.

32. Zaharov $V$. Functional characterization of absolut and Dedekind completion // Bull. Acad. Polon. Sci. Serie sci. math. 1981. V. 29. № 5-6. P. 293-297.

33. Захаров B. K. Связи между расширением Лебега и расширением Бореля первого класса и между соответствующими им прообразами // Изв. АН СССР. Сер. матем. 1990. Т. 54. № 5. С. $928-956$.

34. Hausdorff F. Über halbstetige Functionen und deren Verallgemeinerung // Math. Z. 1915. V. 4. P. 292-309.

35. Sierpin'ski $W$. Sur les fonctions développables en séries absolument conoergentes de fonctions continues // Fund. Math. 1921. V. 2. P. 15-27.

36. Zaharov V. K. Alexandrovian cover and Sierpin'skian extension // Studia Sci. Math. Hung. 1989. V. 24. P. 93-117.

37. Захаров B.K. Связь между полным кольцом частных кольца непрерывных функций, регулярным пополнением и расширениями Хаусдорфа-Серпинского // УМН. 1990. Т. 45. №6. C. $133-134$.

38. Захаров B.K. Расширения кольца непрерывных функций, порожденные регулярным, счетно-делимым и полным кольцами частных, и соответствующие им прообразы // Изв. РАН. Сер. матем. 1995. Т. 59. № 4. С. 15-60.

39. Захаров B. K, Михалев A. В. Проблема общего радоновского представления для произвольного хаусдорфова пространства // Изв. РАН. Сер. матем. 1999. Т. 63. № 5. С. 37-82.

Поступило в редакцию

2.VII.2001 